\title{
A unique pure mechanical system revealing dipole repulsion
}

\author{
June-Haak Ee and Jungil Lee* \\ Department of Physics, Korea University, Seoul 136-713, Korea
}

(Dated: June 16, 2021)

\begin{abstract}
We study multiple elastic collisions of a block and a ball against a rigid wall in one dimension. The complete trajectory of the block is solved as an analytic function of time. Near the turning point of the block the force carried by the ball is proportional to $1 / x^{3}$, where $x$ is the distance between the wall and the block, in the limit that the block is sufficiently heavier than the ball. This is a unique pure mechanical system that reveals dipole-like repulsion.
\end{abstract}




\section{INTRODUCTION}

An elastic collision is a useful mechanical problem with which one can study energy, linearmomentum, and angular-momentum conservation in an explicit way. ${ }^{1}$ While the final state is uniquely determined in a two-body elastic collision in one dimension, there are multiple solutions if three or more particles are involved. The reason is that the two constraints from energy and momentum conservation are not enough to determine the three or more final-state momenta. For example, a unique solution for a Newton's cradle of three or more pendula is obtained only if any two neighboring pendula are separated so that each collision involves only two pendula.

Various studies on such chain collisions have been carried out for the instantaneous contact force, ${ }^{2-7}$ for the spring force, ${ }^{8-11}$ and for the contact Hertz force. ${ }^{7,8,10,12}$ In addition, there are numerous studies of the collisions of aligned balls in one dimension. ${ }^{13-21}$ Harter investigated the velocity gain in such a chain collision. ${ }^{16}$ Patricio studied the effect of the Hertz contact force in detail. ${ }^{7}$ Redner considered the one-dimensional collisions of two cannonballs with a table-tennis ball sandwiched between them to derive a simple relation between that elastic collision and a corresponding billiard system. ${ }^{22}$

In this paper, we consider the multiple collisions of two particles in one dimension against a rigid wall. As is stated above, this problem is, in principle, exactly solvable and we attempt to find the analytic solution to this problem. Many people, who have played or watched table-tennis games, are familiar with this multiple collision. When the server is waiting for the defender, the server often bounces the ball against the table repeatedly with the racket. While the player presses down the ball with the racket, the speed of the ball keeps increasing and one can hear an interesting high-frequency sound near the turning point of the racket.

Whelan et al. studied a similar case under gravity focusing on its chaotic behavior. ${ }^{23}$ Because the main feature of the problem stems from the velocity amplification of the light target against the heavy incident particle, we ignore any external forces other than the contact interaction between any two colliding objects. We construct a simple model system consisting of a block and a rigid wall that sandwich a ball. We derive the analytic expression for the complete trajectory of the block as a function of time. This new analytic solution reveals that, when the ball is sufficiently lighter than the block, the ball mediates a force proportional to $1 / x^{3}$ near the turning point of the block. Here, $x$ is the distance between the 
wall and the block. This emergent dipole interaction is unique in a pure mechanical system. In comparison with a previous study in Ref. ${ }^{24}$ for a case with many balls, our derivation of the $1 / x^{3}$ rule is based on a complete analytic solution with a rigorous error analysis.

This paper is organized as follows. In Sec. II we introduce our model, which simplifies the multiple collision problem of the table-tennis ball. In Sec. III we construct the simultaneous recurrence relation for the velocities of the colliding particles in each collision. Those for the position and time are also given. We determine the complete trajectory of the block as a function of time. In Sec. IV, we investigate the dynamics of the system in the continuum limit, where the block is sufficiently heavier than the ball and the block is near the turning point. Our conclusion is given in Sec. V, followed by the appendices, which contain useful mathematical formulas and some details of calculations.

\section{THE MODEL SYSTEM}

In this section, we define our model system. We restrict ourselves to elastic collision in one spatial dimension and ignore any external forces other than instantaneous contact forces.

As shown in Fig. 1, the model system consists of a block with mass $M$, a ball with mass $m$, and a rigid wall at $x \leq 0$. The unit vector along the positive $x$ axis is given by $\hat{\boldsymbol{x}}$ and the mass ratio is defined by

$$
\alpha=m / M
$$

At time $t=t_{1}=0$ the block hits the ball at $x=x_{1}=L$, where initial velocities of the block and ball are

$$
U_{0}=-U \text { and } \quad u_{0}=0
$$

respectively. After the first collision, the ball bounces against the wall and then they make the next collision. We call $P_{n}\left(t_{n}, x_{n}\right)$ the $n$-th collision point between the ball and the block, where $t_{n}$ and $x_{n}$ are the time and position. The velocities of the block and the ball right after the $n$-th collision are $U_{n}$ and $u_{n}$, respectively. The total number of collisions $N$ is the smallest $n$ that satisfies the condition $U_{n} \geq\left|u_{n}\right|$. We also define the time interval $\Delta t_{n}=t_{n+1}-t_{n}$ between $P_{n}$ and $P_{n+1}$. Useful relations involving the computation of $t_{n}$ using $\Delta t_{n}$ are given in Appendix A. 
The elasticity of the collisions requires the conservation of energy as well as the linear momentum in the $n$-th collision:

$$
\begin{aligned}
M U_{n}-m u_{n} & =M U_{n+1}+m u_{n+1} \\
\frac{1}{2} M U_{n}^{2}+\frac{1}{2} m u_{n}^{2} & =\frac{1}{2} M U_{n+1}^{2}+\frac{1}{2} m u_{n+1}^{2} .
\end{aligned}
$$

Note that the sign of $u_{n}$ is flipped in the first equation because the ball has bounced against the wall. With the initial conditions in Eq. (2), one can solve $U_{n}$ and $u_{n}$ recursively. The first two pairs of the solutions are

$$
\begin{aligned}
& \left(\begin{array}{l}
U_{1} \\
u_{1}
\end{array}\right)=-\frac{U}{1+\alpha}\left(\begin{array}{c}
1-\alpha \\
2
\end{array}\right), \\
& \left(\begin{array}{l}
U_{2} \\
u_{2}
\end{array}\right)=-\frac{U}{(1+\alpha)^{2}}\left(\begin{array}{c}
1-6 \alpha+\alpha^{2} \\
4(1-\alpha)
\end{array}\right) .
\end{aligned}
$$

Based on Eqs. (4), we classify the ranges of the mass ratio $\alpha$ that determine the value of $N$.

- If $\alpha \geq 3$, then $N=1$ because $U_{1} \geq-u_{1}$. The block bounces back after the first collision and they never collide again. At $\alpha=3, U_{1}=-u_{1}=\frac{1}{2} U$.

- If $1 \leq \alpha<3$, then $N=2$ because $U_{1}<-u_{1}$ and $U_{2}>u_{2} \geq 0$. At $\alpha=1, U_{1}=0$, $u_{1}=-U, U_{2}=U$, and $u_{2}=0$.

- If $\alpha<1$, then $N \geq 2$. Especially, for an $\alpha<5-2 \sqrt{5}, N \geq 3$. At $\alpha=5-2 \sqrt{5}$, $U_{2}=-u_{2}$.

In the remainder of this paper, we consider the case $0<\alpha<1$ that involves multiple collisions.

\section{ANALYTIC SOLUTIONS}

In this section, we determine the point $P_{n}\left(t_{n}, x_{n}\right)$ of the $n$-th collision between the ball and block, and the velocities $U_{n}$ and $u_{n}$ immediately after the collision. 


\section{A. Computation of $U_{n}$ and $u_{n}$}

We can reduce the recurrence relations for the velocities in Eq. (3) into the form

$$
\left(\begin{array}{c}
U_{n+1} \\
u_{n+1}
\end{array}\right)=A\left(\begin{array}{l}
U_{n} \\
u_{n}
\end{array}\right),
$$

where the $2 \times 2$ matrix $A$ is defined by

$$
A=\frac{1}{1+\alpha}\left(\begin{array}{cc}
1-\alpha & -2 \alpha \\
2 & 1-\alpha
\end{array}\right) .
$$

Applying Eq. (5) recursively, we determine $U_{n}$ and $u_{n}$ :

$$
\left(\begin{array}{c}
U_{n} \\
u_{n}
\end{array}\right)=A^{n}\left(\begin{array}{l}
U_{0} \\
u_{0}
\end{array}\right) .
$$

Although our initial conditions are given in Eq. (2), the relation (7) is valid for any initial values of $U_{0}$ and $u_{0}$.

The computation of $U_{n}$ and $u_{n}$ is rather tedious and we summarize the calculation in Appendix B. The results are

$$
\left(\begin{array}{l}
U_{n} \\
u_{n}
\end{array}\right)=-U\left(\begin{array}{c}
\cos n \theta \\
\frac{1}{\sqrt{\alpha}} \sin n \theta
\end{array}\right),
$$

where the parameter $\theta$ is related to the mass ratio $\alpha$ as

$$
\tan \frac{\theta}{2}=\sqrt{\alpha} .
$$

Let us interpret the results in Eq. (8). Because the total kinetic energy is the sum of quadratic functions of $U_{n}$ and $u_{n}$, the conservation of the total kinetic energy implies that $U_{n}$ and $u_{n}$ can be parametrized as a cosine and a sine of a common phase. The phase increases as a multiple of an elementary phase $\theta$ because the two eigenvalues of the non-Hermitian matrix $A$ are a unimodulus complex number $e^{i \theta}$ and its complex conjugate. According to these results, the block slows down to reach the turning point around $n \theta \approx \frac{\pi}{2}$, where $\left|u_{n}\right|$ becomes the maximum. The maximum value for $\left|u_{n}\right|$ is larger than the initial speed $U$ of the block with the enhancement factor $1 / \sqrt{\alpha}$, if $0<\alpha<1$. From that moment to the region $n \theta \approx \pi$, the block bounces back and the ball loses the kinetic energy. If $n=N$, then the velocity 
$-u_{n}$ of the ball after the bounce against the wall must satisfy the constraint $U_{n} \geq-u_{n}$. Therefore, $N$ must be the smallest integer that satisfies

$$
U_{N}+u_{N} \geq 0 \quad \text { and } \quad N>\frac{\pi}{2 \theta}
$$

\section{B. Computation of $x_{n}$}

The recurrence relation of $x_{n}$ is derived in Eq. (A2) of Appendix A:

$$
\frac{x_{n+1}}{x_{n}}=\left(u_{n}-U_{n}\right) /\left(u_{n}+U_{n}\right) .
$$

By making use of Eqs. (8) and (9) and the elementary trigonometric identity $\sin \alpha \cos \beta \pm$ $\cos \alpha \sin \beta=\sin (\alpha \pm \beta)$, we find that

$$
\frac{x_{n+1}}{x_{n}}=\frac{\sin \left(n-\frac{1}{2}\right) \theta}{\sin \left(n+\frac{1}{2}\right) \theta} .
$$

With the initial condition $x_{1}=L$, we find the analytic expression for $x_{n}$ as

$$
x_{n}=x_{1} \prod_{k=1}^{n-1} \frac{x_{k+1}}{x_{k}}=\frac{L \sin \frac{\theta}{2}}{\sin \left(n-\frac{1}{2}\right) \theta} .
$$

The analytic expression for $x_{n}$ is new. From this compact expression, it is easy to see that $x_{n}$ decreases to reach the minimum value $x_{\min }$ near $n \approx \frac{1}{2}\left(\frac{\pi}{\theta}-1\right)$, where the denominator reaches its maximum. The turning point $x_{\min }$ is reached at $n=N_{1}$ and the last collision is made at $x_{N}$.

\section{Computation of $N_{1}$ and $N$}

From Eq. (13), we can determine that $N_{1}$ is

$$
N_{1}=\left[\frac{\pi}{2 \theta}\right]_{+},
$$

where $[x]_{+}$is the smallest integer that is greater than or equal to $x$. For a small $\alpha, \theta \approx 2 \sqrt{\alpha}$ and therefore $N_{1} \approx\left[\frac{\pi}{4 \sqrt{\alpha}}\right]_{+}$. In analogy to the derivation of Eq. (13), we can simplify $U_{n}+u_{n}$ as

$$
U_{n}+u_{n}=-\frac{U \sin \left(n+\frac{1}{2}\right) \theta}{\sin \frac{\theta}{2}}
$$


Substituting Eq. (15) into Eq. (10), we find that

$$
N=\left[\frac{\pi}{\theta}-\frac{1}{2}\right]_{+} \approx\left[\frac{\pi}{2 \sqrt{\alpha}}-\frac{1}{2}\right]_{+},
$$

where the final expression is valid for small $\alpha$. This result is consistent with Eq. (6) of Ref. ${ }^{22}$ with the uncertainty \pm 1 . The quantity $N_{\max }$ of that reference is approximately equal to $2 N$ because, in that reference, the collision between the ball and the wall is also counted.

In Fig. 2, we show the total number of collisions $N$ as a function of $\alpha$. The results in the figure are consistent with classification given at the end of Sec. II. The discontinuities of $N$ are at $\alpha=\alpha_{i}$, where $\alpha_{i}$ is the minimum value of $\alpha$ that satisfies $N=i$. The first two values of $\alpha_{i}$ are $\alpha_{1}=3$ and $\alpha_{2}=5-2 \sqrt{5}$.

\section{Computation of $t_{n}$}

We have determined $U_{n}, u_{n}$, and $x_{n}$ for all $n=1, \cdots, N$. In this section, we use these results to compute the collision times $t_{n}$. The computation of the time interval $\Delta t_{n}$ is given in Appendix C. By making use of Eq. (A4), we sum the time intervals $\Delta t_{n}$ in Eq. (C3) to find

$$
t_{n}=\tau\left[1-\frac{\tan \frac{\theta}{2}}{\tan \left(n-\frac{1}{2}\right) \theta}\right],
$$

where $\tau$ is the time interval for the block to reach the wall when the ball is absent $(\alpha=0)$ :

$$
\tau=L / U
$$

This analytic expression (17) for $t_{n}$ is new.

The complete trajectory of the block is

$$
x(t)=x_{n}+U_{n}\left(t-t_{n}\right), \quad t_{n} \leq t \leq t_{n+1},
$$

where $x_{n}, U_{n}$, and $t_{n}$ are given in Eqs. (13), (8), and (17), respectively. In Fig. 3, we plot the trajectory $x(t)$ of the block as a collection of straight line segments. The vertices represent the collision points $P_{n}\left(t_{n}, x_{n}\right)$. The point on the vertical axis represents the initial collision point $P_{1}(0, L)$. The turning point is $P_{N_{1}}\left(t_{\min }, x_{\min }\right)$. When $\alpha=0.25(0.1)$, we have $N_{1}=2$ (3), and $N=3(5)$. The $x$-t plot is convex-downward because every impact on the the block is along the positive $x$ axis. 


\section{E. Computation of $x_{\min }$ and $t_{\text {min }}$}

Next we find the turning point $x_{\min }=x_{N_{1}}$ and corresponding time $t_{\min }=t_{N_{1}}$. According to Eq. (14), $\left(N_{1}-\frac{1}{2}\right) \theta$ is bounded by

$$
\frac{1}{2}(\pi-\theta) \leq\left(N_{1}-\frac{1}{2}\right) \theta<\frac{1}{2}(\pi+\theta) .
$$

Therefore, $\cos \frac{\theta}{2} \leq \sin \left(N_{1}-\frac{1}{2}\right) \theta \leq 1$. Substituting this constraint into Eq. (13) with $n=N_{1}$, we can determine the range of $x_{\min }$ as $L \sin \frac{\theta}{2} \leq x_{N_{1}} \leq L \tan \frac{\theta}{2}$. With the values for $\sin \frac{\theta}{2}$ and $\tan \frac{\theta}{2}$ in Table I, we obtain

$$
\frac{L \sqrt{\alpha}}{\sqrt{1+\alpha}} \leq x_{\min } \leq L \sqrt{\alpha}
$$

When $\alpha$ is small, $x_{\min } \approx L \sqrt{\alpha}$. From the range in Eq. (20) we find that $\cot \frac{\theta}{2} \leq \tan \left(N_{1}-\right.$ $\left.\frac{1}{2}\right) \theta<\infty$ or $-\infty<\tan \left(N_{1}-\frac{1}{2}\right) \theta<-\cot \frac{\theta}{2}$. Therefore, considering both cases, we obtain

$$
\tau(1-\alpha) \leq t_{\min }<\tau(1+\alpha)
$$

where we used $\tan ^{2} \frac{1}{2} \theta=\alpha$. The errors of $x_{\min }$ and $t_{\min }$ are $\pm \frac{1}{2} L \sqrt{\alpha}(1-1 / \sqrt{1+\alpha})$ and $\pm \alpha \tau$, respectively.

It is interesting to notice that when $\alpha$ is sufficiently small, the time interval $t_{\min }$ for the block to reach the turning point is approximately the same as $\tau$, which is the time spent for the block to hit the wall when the ball is missing.

\section{CONTINUUM LIMIT}

According to Eq. (C1), $\Delta t_{n}$ becomes small when $\alpha$ is sufficiently small and $n \approx N_{1}$, where $\sin \left(n-\frac{1}{2}\right) \theta \approx 1$. This does not require that $\alpha \rightarrow 0^{+}$. We call this the continuum limit. In this limit, both $x_{n}$ and $t_{n}$ can be treated to be continuous variables.

In this section, we investigate the dynamics of the system in the continuum limit. As the first step, we express the collision point $x_{n}$ in terms of $t_{n}$. Next, we introduce a differentiable function $\bar{x}(t)$ that may represent the trajectory of the block in the limit. By making use of the kinetic energy conservation, we interpret the kinetic energy of the ball as the potential energy of the block. Expressing the kinetic energy of the ball in terms of the differentiable function $\bar{x}(t)$, we compute the force on the block in the continuum limit. 


\section{A. Relation between $x_{n}$ and $t_{n}$}

We observe that both $x_{n}$ in Eq. (13) and $t_{n}$ in Eq. (17) depend on $n$ explicitly. In this section, we find the analytic expression for $x_{n}$ as a function of $t_{n}$ by eliminating the explicit $n$ dependence.

From Eqs. (13) and (17), we have

$$
\begin{aligned}
& x_{n} \cos \left(n-\frac{1}{2}\right) \theta=L \cdot \frac{1-t_{n} / \tau}{\sqrt{1+\alpha}}, \\
& x_{n} \sin \left(n-\frac{1}{2}\right) \theta=L \sqrt{\frac{\alpha}{1+\alpha}},
\end{aligned}
$$

where $\sin \frac{\theta}{2}$ and $\tan \frac{\theta}{2}$ are replaced with the values listed in Table I. By making use of the fact that $\sin \left(n-\frac{1}{2}\right) \theta \geq 0$ for all $n \leq N$, we can express $x_{n}$ as a function of $t_{n}$ :

$$
x_{n}=\frac{L}{\sqrt{1+\alpha}} \sqrt{\left(1-t_{n} / \tau\right)^{2}+\alpha},
$$

where we used relations in Eq. (23) and Table I.

In Appendix $\mathrm{D}$, we have shown that the complete trajectory $x(t)$ of the block is always bounded by

$$
f(t) \leq x(t) \leq g(t)
$$

where the differentiable functions $f(t)$ and $g(t)$ are defined by

$$
\begin{aligned}
f(t) & =\frac{L}{\sqrt{1+\alpha}} \sqrt{(1-t / \tau)^{2}+\alpha}, \\
g(t) & =L \sqrt{(1-t / \tau)^{2}+\alpha} .
\end{aligned}
$$

As shown in Fig. 3, the lower bound $f(t)$ passes every collision point $P_{n}$ and the upper bound $g(t)$ is tangent to every line segment $\overline{P_{n} P_{n+1}}$.

In the continuum limit, it is convenient to use the arithmetic average $\bar{x}(t)=\frac{1}{2}[g(t)+f(t)]$ to represent the trajectory of the block with the uncertainty $\delta \bar{x}(t)=\frac{1}{2}[g(t)-f(t)]$ :

$$
\begin{aligned}
\bar{x}(t) & =\mathcal{N} L \sqrt{(1-t / \tau)^{2}+\alpha}, \\
\delta \bar{x}(t) & =\Delta L \sqrt{(1-t / \tau)^{2}+\alpha},
\end{aligned}
$$

where $\mathcal{N}=\frac{1}{2}\left[1+(1+\alpha)^{-\frac{1}{2}}\right]$ and $\Delta=\frac{1}{2}\left[1-(1+\alpha)^{-\frac{1}{2}}\right]$. In the limit $\alpha \rightarrow 0^{+}$, all of the curves $\bar{x}(t), f(t)$, and $g(t)$ collapse into $x(t)$. In addition,

$$
\lim _{\alpha \rightarrow 0^{+}} x(t)=L|1-t / \tau|,
$$


which is the case that the ball is absent.

In the continuum limit we can compute the velocity and the acceleration of the block using $\bar{x}(t)$ :

$$
\begin{aligned}
& \dot{\bar{x}}(t)=-\frac{\mathcal{N} U(1-t / \tau)}{\sqrt{(1-t / \tau)^{2}+\alpha}}, \\
& \ddot{\bar{x}}(t)=\frac{\alpha \mathcal{N} U / \tau}{\left[(1-t / \tau)^{2}+\alpha\right]^{3 / 2}} .
\end{aligned}
$$

The values for $\bar{x}, \dot{\bar{x}}$, and $\ddot{\bar{x}}$ must be understood as the time-averaged values. The uncertainties of $\dot{\bar{x}}(t)$ and $\ddot{\bar{x}}(t)$ can be obtained by replacing $\mathcal{N}$ with $\Delta$.

\section{B. Kinetic energy of the block}

Based on Eqs. (29a) and (27a), we can determine the kinetic energy $K$ of the block in terms of $t$ or $x$ as

$$
\begin{aligned}
K(t) & =K_{0} \frac{\mathcal{N}^{2}(1-t / \tau)^{2}}{(1-t / \tau)^{2}+\alpha}, \\
K(x) & =K_{0} \mathcal{N}^{2}\left[1-\frac{\alpha \mathcal{N}^{2} L^{2}}{x^{2}}\right],
\end{aligned}
$$

where $K_{0}=\frac{1}{2} M U^{2}$ is the initial kinetic energy, which can be approximated as $K_{0} \approx K(t=$ $-\infty)$. For a small $\alpha$, the relative error of the kinetic energy is $\pm \frac{1}{2} \alpha$.

\section{Potential energy of the block}

We recall that the total kinetic energy is conserved during the whole process. Therefore, the sum of the kinetic energies of the block $(K)$ and the ball $(\Phi)$ must be

$$
K+\Phi=K_{0}
$$

We interpret the kinetic energy $\Phi$ of the ball as the potential energy of the block. By making use of Eqs. (30a), (30b), and (31), we find that

$$
\begin{aligned}
& \Phi(t)=K_{0}\left[1-\frac{\mathcal{N}^{2}(1-t / \tau)^{2}}{(1-t / \tau)^{2}+\alpha}\right] \\
& \Phi(x)=K_{0}\left[1-\mathcal{N}^{2}+\frac{\alpha \mathcal{N}^{4} L^{2}}{x^{2}}\right]
\end{aligned}
$$


Next we compute the force,

$$
F(x)=-\frac{d \Phi(x)}{d x}=\frac{\alpha \mathcal{N}^{4} M U}{\tau} \cdot \frac{L^{3}}{x^{3}} .
$$

The force on the block has the same position dependence as an electric dipole interaction: $F(x) \sim 1 / x^{3}$. In addition, $x=x_{\min }$ when $t=\tau$. Therefore, the duration of time to reach

$x_{\min }$ is the same as the case when the ball is absent. As is stated earlier, the derivation of the $1 / x^{3}$ rule originates from the analytic solution in Eq. (27), which is in contrast to the approach in Ref. ${ }^{24}$.

We can compute $x_{\min }$ in the continuum limit. Setting $\Phi\left(x_{\min }\right)=K_{0}$, we find that

$$
\begin{aligned}
& x_{\min }=\mathcal{N} L \sqrt{\alpha}, \\
& t_{\min }=\tau,
\end{aligned}
$$

These values are consistent with Eqs. (21) and (22) within errors.

\section{CONCLUSION}

We have considered the one-dimensional elastic collisions of a ball and a block against a rigid wall. The initial state of the ball is $u_{0}=0$ at $x=L$ and that of the block is $U_{0}=-U$. The trajectory $x(t)$ of the block is completely determined in an analytic form. The analytic expression for the total number $N$ of collisions between the block and the ball was also derived. The turning point of the block is $\approx L \sqrt{\alpha}$ at $t \approx \tau=L / U$, which is the time interval for the block to hit the wall when the ball is absent. Here $\alpha$ is the ratio of the mass of the ball to the mass of the block.

In the continuum limit where $\alpha$ is small and $x(t)$ is near the turning point, the trajectory can be approximated as a differentiable function $\bar{x}(t)$ in Eq. (27a). Because the total kinetic energy is conserved in this system, one can think of the kinetic energy of the ball as the potential energy of the block. Based on this idea, we have computed the force on the block and found that the force is proportional to $1 / x^{3}$. It is remarkable that this is a unique pure mechanical system that reveals repulsive dipole-like interaction. 


\section{Appendix A: Useful Formulas to compute $t_{n}$}

The time interval between $P_{n}$ and $P_{n+1}$ is defined by $\Delta t_{n}=t_{n+1}-t_{n}$ which can be expressed in terms of $U_{n}$ or $u_{n}$ as

$$
\Delta t_{n}=\frac{x_{n+1}-x_{n}}{U_{n}}=\frac{s_{n}}{\left|u_{n}\right|}
$$

where $s_{n}=x_{n}+x_{n+1}$ is the distance that the ball travels between $P_{n}$ and $P_{n+1}$. Assuming $u_{n}<0$, we find that

$$
x_{n+1}=x_{n}\left(u_{n}-U_{n}\right) /\left(u_{n}+U_{n}\right) .
$$

Substituting Eq. (11) into Eq. (A1), we can express $\Delta t_{n}$ as

$$
\Delta t_{n}=-\frac{2 x_{n}}{U_{n}+u_{n}} .
$$

The $n$-th collision time $t_{n}$ can be computed as

$$
t_{n}=\sum_{k=1}^{n-1} \Delta t_{k}
$$

\section{Appendix B: Computation of $U_{n}$ and $u_{n}$}

In this section, we solve Eq. (7),

$$
\left(\begin{array}{l}
U_{n} \\
u_{n}
\end{array}\right)=A^{n}\left(\begin{array}{l}
U_{0} \\
u_{0}
\end{array}\right)
$$

where the matrix $A$ is defined in Eq. (6),

$$
A=\frac{1}{1+\alpha}\left(\begin{array}{cc}
1-\alpha & -2 \alpha \\
2 & 1-\alpha
\end{array}\right)
$$

We make a transformation diagonalizing $A$ such that

$$
\left(\begin{array}{c}
W_{n} \\
w_{n}
\end{array}\right)=R^{-1}\left(\begin{array}{l}
U_{n} \\
u_{n}
\end{array}\right), \quad R^{-1} A R=\left(\begin{array}{cc}
\lambda_{1} & 0 \\
0 & \lambda_{2}
\end{array}\right),
$$

where $R$ is a $2 \times 2$ matrix and $\lambda_{i}$ 's are the eigenvalues of the matrix $A$. Then, $U_{n}$ and $u_{n}$ are

$$
\left(\begin{array}{c}
U_{n} \\
u_{n}
\end{array}\right)=R\left(\begin{array}{c}
\lambda_{1}^{n} W_{0} \\
\lambda_{2}^{n} w_{0}
\end{array}\right), \quad\left(\begin{array}{l}
W_{0} \\
w_{0}
\end{array}\right)=R^{-1}\left(\begin{array}{c}
-U \\
0
\end{array}\right) .
$$


The $i$-th column vector $R_{i}$ of $R$ satisfies the equation,

$$
A R_{i}=\lambda_{i} R_{i}
$$

for $i=1$ and 2 . The corresponding secular equation $\operatorname{det}\left[A-\lambda_{i} \mathbb{1}\right]=0$, where $\mathbb{1}$ is the $2 \times 2$ identity matrix, is

$$
\alpha\left(1+\lambda_{i}\right)^{2}+\left(1-\lambda_{i}\right)^{2}=0
$$

Solving this equation, we obtain

$$
\begin{aligned}
& \lambda_{1}=e^{+i \theta}, \quad R_{1}=\left(\begin{array}{c}
1 \\
-i / \sqrt{\alpha}
\end{array}\right), \\
& \lambda_{2}=e^{-i \theta}, \quad R_{2}=\left(\begin{array}{c}
1 \\
+i / \sqrt{\alpha}
\end{array}\right),
\end{aligned}
$$

where the parameter $\theta$ is related to the mass ratio $\alpha$ as

$$
\tan \frac{\theta}{2}=\sqrt{\alpha}
$$

In Sec. IV of Ref. ${ }^{23}$, the authors considered the collisions of two balls near the ground, which can be compared to our results by neglecting gravity. The matrix $A$ in Eq. (6) is equivalent to the matrix $M$ in Eq. (13) of Ref. ${ }^{23}$. The elements in the second column have opposite signs because of the difference in the definition of $u_{n}$. The eigenvalues $\lambda_{i}$ in Eq. (B7) and the parameter $\theta$ in Eq. (9) are consistent with those in Eq. (15) of Ref. ${ }^{23}$.

Here, we consider only the case $0<\alpha<1$. Note that $\theta=0$ for $\alpha=0,0<\theta<\frac{\pi}{2}$ for $0<\alpha<1$, and $\theta=\frac{\pi}{2}$ for $\alpha=1$. In the second and third columns of Table I, we list the values for trigonometric functions for $\frac{1}{2} \theta$ and $\theta$, respectively. Now we determine $R$ and $R^{-1}$ as

$$
R=\left(\begin{array}{cc}
1 & 1 \\
-i / \sqrt{\alpha} & i / \sqrt{\alpha}
\end{array}\right), \quad R^{-1}=\frac{1}{2}\left(\begin{array}{cc}
1 & i \sqrt{\alpha} \\
1 & -i \sqrt{\alpha}
\end{array}\right) .
$$

Substituting Eq. (B9) into Eq. (B4), we finally determine the velocities $U_{n}$ and $u_{n}$ as functions of $n$ and $\alpha$ :

$$
\left(\begin{array}{l}
U_{n} \\
u_{n}
\end{array}\right)=-U\left(\begin{array}{c}
\cos n \theta \\
\frac{1}{\sqrt{\alpha}} \sin n \theta
\end{array}\right)
$$




\section{Appendix C: Computation of the time interval $\Delta t_{n}$}

The time interval $\Delta t_{n}=t_{n+1}-t_{n}$ can be computed by substituting $x_{n}$ in Eq. (13) and $U_{n}+u_{n}$ in Eq. (15) into Eq. (A3).

$$
\Delta t_{n}=\frac{2 \tau \sin ^{2} \frac{\theta}{2}}{\sin \left(n-\frac{1}{2}\right) \theta \sin \left(n+\frac{1}{2}\right) \theta},
$$

where $\tau$ is the time interval for the block to reach the wall when the ball is absent $(\alpha=0)$,

that is, $\tau=L / U$. Substituting $\alpha=\left(n-\frac{1}{2}\right) \theta$ and $\beta=\left(n+\frac{1}{2}\right) \theta$ into the following trigonometric identity,

$$
\frac{\sin (\beta-\alpha)}{\sin \alpha \sin \beta}=\cot \alpha-\cot \beta
$$

we can simplify Eq. (C1) as

$$
\Delta t_{n}=\tau\left[\frac{\tan \frac{\theta}{2}}{\tan \left(n-\frac{1}{2}\right) \theta}-\frac{\tan \frac{\theta}{2}}{\tan \left(n+\frac{1}{2}\right) \theta}\right] .
$$

\section{Appendix D: Determination of the functions $f(t)$ and $g(t)$}

The complete trajectory of the block $x(t)$ is a set of line segments that connects consecutive collision point such that $x\left(t_{n}\right)=x_{n}$, where

$$
x_{n}=\frac{L}{\sqrt{1+\alpha}} \sqrt{\left(1-t_{n} / \tau\right)^{2}+\alpha} .
$$

We introduce a differentiable function,

$$
f(t)=\frac{L}{\sqrt{1+\alpha}} \sqrt{(1-t / \tau)^{2}+\alpha}
$$

that passes every collision point $P_{n}$ and is convex downward. Therefore, at any time $t \in$ $\left[0, t_{N}\right], f(t) \leq x(t)$, where the equality holds only at $t=t_{n}$ for $n=1, \cdots, N$.

The time derivative of Eq. (D2) is

$$
\dot{f}(t)=-\frac{U}{\sqrt{1+\alpha}} \frac{1-t / \tau}{\sqrt{(1-t / \tau)^{2}+\alpha}} .
$$

By making use of the special values of the trigonometric functions in Table I, we find that

$$
\dot{f}\left(t_{n}\right)=-U \cos \frac{\theta}{2} \cos \left(n-\frac{1}{2}\right) \theta=\frac{1}{2}\left(U_{n-1}+U_{n}\right),
$$

where we have used $\cos (\alpha+\beta)+\cos (\alpha-\beta)=2 \cos \alpha \cos \beta$ for $\alpha=\left(n-\frac{1}{2}\right) \theta$ and $\beta=\frac{1}{2} \theta$. 
If there exists a curve $g(t)$ that is tangent to every line segment $\overline{P_{n} P_{n+1}}$ at $t_{n}^{\prime} \in\left[t_{n}, t_{n+1}\right]$ and convex downward, then $g(t)$ is an upper bound of $x(t)$ for all $t \in\left[0, t_{N}\right]$. We require

$$
\dot{g}\left(t_{n}^{\prime}\right)=U_{n}=-U \cos n \theta,
$$

and we set $x_{n}^{\prime}=g\left(t_{n}^{\prime}\right)$. According to Eq. (D4), $\dot{g}\left(t_{n}^{\prime}\right)=\dot{f}\left(t_{n+\frac{1}{2}}\right) / \cos \frac{\theta}{2}$. Therefore, we find that

$$
\begin{aligned}
\dot{g}(t) & =-\frac{U(1-t / \tau)}{\sqrt{(1-t / \tau)^{2}+\alpha}}, \\
t_{n}^{\prime} & =t_{n+\frac{1}{2}}=\tau(1-\sqrt{\alpha} \cot n \theta), \\
x_{n}^{\prime} & =x\left(t_{n+\frac{1}{2}}\right)=\frac{L \sin \frac{\theta}{2}}{\sin n \theta} .
\end{aligned}
$$

Note that $t_{n} \leq t_{n}^{\prime} \leq t_{n+1}$ and $\dot{g}\left(t_{n}\right)=-U \cos \left(n-\frac{1}{2}\right) \theta$. Integrating $\dot{g}(t)$ in Eq. (D6a) over $t$ and imposing the boundary condition (D6c), we obtain

$$
g(t)=L \sqrt{(1-t / \tau)^{2}+\alpha} .
$$

As a result, the trajectory $x(t)$ is bounded by

$$
f(t) \leq x(t) \leq g(t)
$$

for any $t \in\left[0, t_{N}\right]$. The left and right equalities hold at $t=t_{n}$ and at $t=t_{n}^{\prime}=t_{n+\frac{1}{2}}$, respectively, for all $n$. In Fig. 3, we show the trajectories of the block $x(t)$ at $\alpha=0.25$ and $\alpha=0.1$. As shown in this figure, the lower bound $f(t)$ passes every collision point $P_{n}$ and the upper bound $g(t)$ is tangent to every line segment $\overline{P_{n} P_{n+1}}$.

\section{Acknowledgments}

We thank U-Rae Kim for careful reading of the manuscript and useful comments. JL expresses his gratitude to the members of Korea University Board of Interdisciplinary Communication for an enjoyable discussion that motivated the work presented here. The work of JL was supported by Mid-career Research Program through the NRF grant funded by the MEST (2011-0027559).

\footnotetext{
* Electronic address: jungil@hep.anl.gov
} 
1 See, for example, C. Kittel, W. D. Knight, and M. A. Ruderman, Mechanics, Berkeley Physics Course, Vol. 1 (McGraw-Hill, New York, 1973), 2nd ed.; D. Halliday and R. Resnick, Fundamentals of Physics (Wiley, New York, 1981), 2nd ed. extended version.

2 J. V. Kline, "The Case of the Counting Balls," Am. J. Phys. 28, 102-103 (1960).

3 J. D. Kerwin, "Velocity, Momentum, and Energy Transmissions in Chain Collisions," Am. J. Phys. 40, 1152-1157 (1972).

4 J. S. Huebner and T. L. Smith, "Multi-Ball Collisions," Phys. Teach. 30, 46-47 (1992).

5 T. J. Murphy, "Dynamics of Hard Rods in One Dimension," J. Stat. Phys. 74, 889-901 (1994).

6 H. Erlichson, "Newton's Strange Collisions," Phys. Teach. 33, 169-171 (1996).

7 P. Patricío, "The Hertz contact in chain elastic collisions," Am. J. Phys. 72, 1488-1491 (2004).

8 S. Chapman, "Misconception Concerning the Dynamics of the Impact Ball Apparatus," Am. J. Phys. 28, 705-711 (1960).

9 L. Flansburg and K. Hudnut, "Dynamic solutions for linear elastic collisions," Am. J. Phys. 47, 911-914 (1979).

10 E. J. Hinch and S. Saint-Jean, "The fragmentation of a line of balls by an impact," Proc. R. Soc. Lond. A 455, 3201-3220 (1999).

11 F. Herrmann and P. Schmälzle, "Simple explanation of a well-known collision experiment," Am. J. Phys. 49, 761-764 (1981).

12 R. Hessel, A. C. Perinotto, R. A. M. Alfaro, and A. A. Freschi, "Force-versus-time curves during collisions between two identical steel balls," Am. J. Phys. 74, 176-179 (2006).

13 H. B. Lemon, "An Almost Forgotten Case of Elastic Impact," Am. Phys. Teach. 3, 36-36 (1935).

14 W. W. Sleator, "Impact of Elastic Spheres," Am. J. Phys. 5, 284-285 (1937).

15 W. R. Mellen, "Superball Rebound Projectiles," Am. J. Phys. 36, 845-845 (1968).

16 W. G. Harter, "Velocity Amplification in Collision Experiments Involving Superballs," Am. J. Phys. 39, 656-663 (1971).

17 G. Stroink, "Superball problem," Phys. Teach. 21, 466-466 (1983).

18 I. Bruce, "A one-dimension collision experiment," Am. J. Phys. 58, 696-696 (1990).

19 W. R. Mellen, "Aligner for Elastic Collisions of Dropped Balls," Phys. Teach. 33, 56-57 (1995).

20 R. Cross, "Vertical bounce of two vertically aligned balls," Am. J. Phys. 75, 1009-1016 (2007).

21 P. Müller and T. Pöschel , "Two-ball problem revisited: Limitations of event-driven modeling," Phys. Rev. E 83, 041304-041304 (2011). 
22 S. Redner, "A billiard-theoretic approach to elementary one-dimensional elastic collisions," Am. J. Phys. 72, 1492-1498 (2004).

23 N. D. Whelan, D. A. Goodings, and J. K. Cannizzo, "Two balls in one dimension with gravity," Phys. Rev. A 42, 742-754 (1990).

24 Y. G. Sinai, "Dynamics of a heavy particle surrounded by a finite number of light particles," Theor. Math. Phys. 121, 1351-1357 (1991). 


\section{Tables}

\begin{tabular}{lccc}
\hline \hline$f(x) \backslash x$ & $\frac{1}{2} \theta$ & $\theta$ & $\left(n-\frac{1}{2}\right) \theta$ \\
\hline $\cos x$ & $\frac{1}{\sqrt{1+\alpha}}$ & $\frac{1-\alpha}{1+\alpha}$ & $\frac{1-t_{n} / \tau}{\sqrt{\left(1-t_{n} / \tau\right)^{2}+\alpha}}$ \\
$\sin x$ & $\frac{\sqrt{\alpha}}{\sqrt{1+\alpha}}$ & $\frac{2 \sqrt{\alpha}}{1+\alpha}$ & $\frac{\sqrt{\alpha}}{\sqrt{\left(1-t_{n} / \tau\right)^{2}+\alpha}}$ \\
$\tan x$ & $\sqrt{\alpha}$ & $\frac{2 \sqrt{\alpha}}{1-\alpha}$ & $\frac{\sqrt{\alpha}}{1-t_{n} / \tau}$ \\
\hline \hline
\end{tabular}

TABLE I: The values for $\cos x, \sin x$, and $\tan x$ for $x=\frac{1}{2} \theta, \theta$, and $\left(n-\frac{1}{2}\right) \theta$. The values at $x=n \theta$ and $\left(n+\frac{1}{2}\right) \theta$ can be obtained by using the identities such as $\cos (x \pm \delta)=\cos x \cos \delta \mp \sin x \sin \delta$ and $\sin (x \pm \delta)=\sin x \cos \delta \pm \cos x \sin \delta$.

\section{Figures}

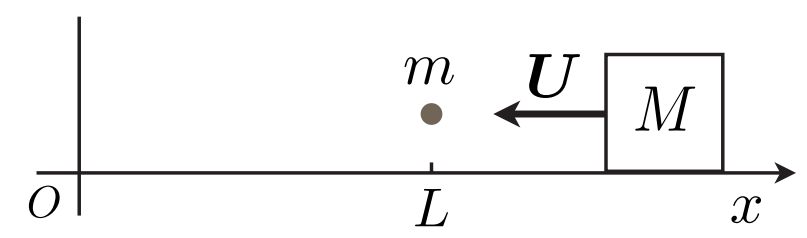

FIG. 1: The initial condition of the model system. 


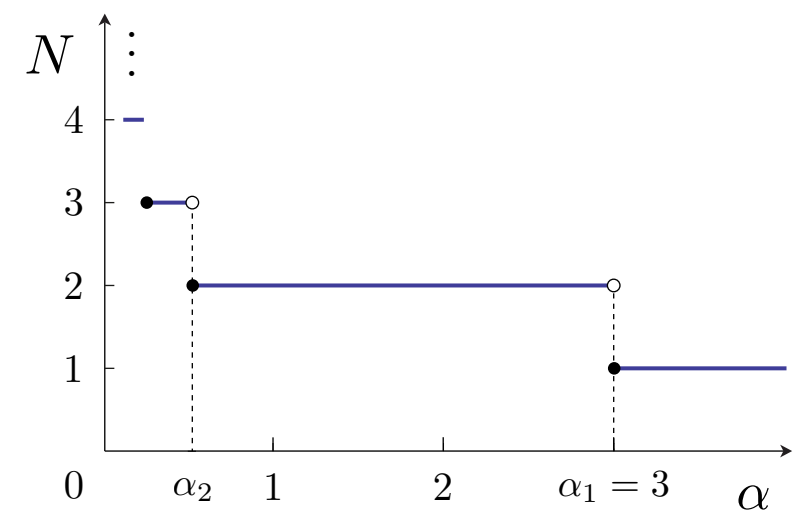

FIG. 2: The total number of collision $N$ as a function of the mass ratio $\alpha . \alpha_{1}=3$ and $\alpha_{2}=5-2 \sqrt{5}$ are the minimum values of $\alpha$ to have $N=1$ and $N=2$, respectively. As $\alpha \rightarrow 0^{+}, N \rightarrow\left[\frac{\pi}{2 \sqrt{\alpha}}-\frac{1}{2}\right]_{+}$ which diverges to infinity. 

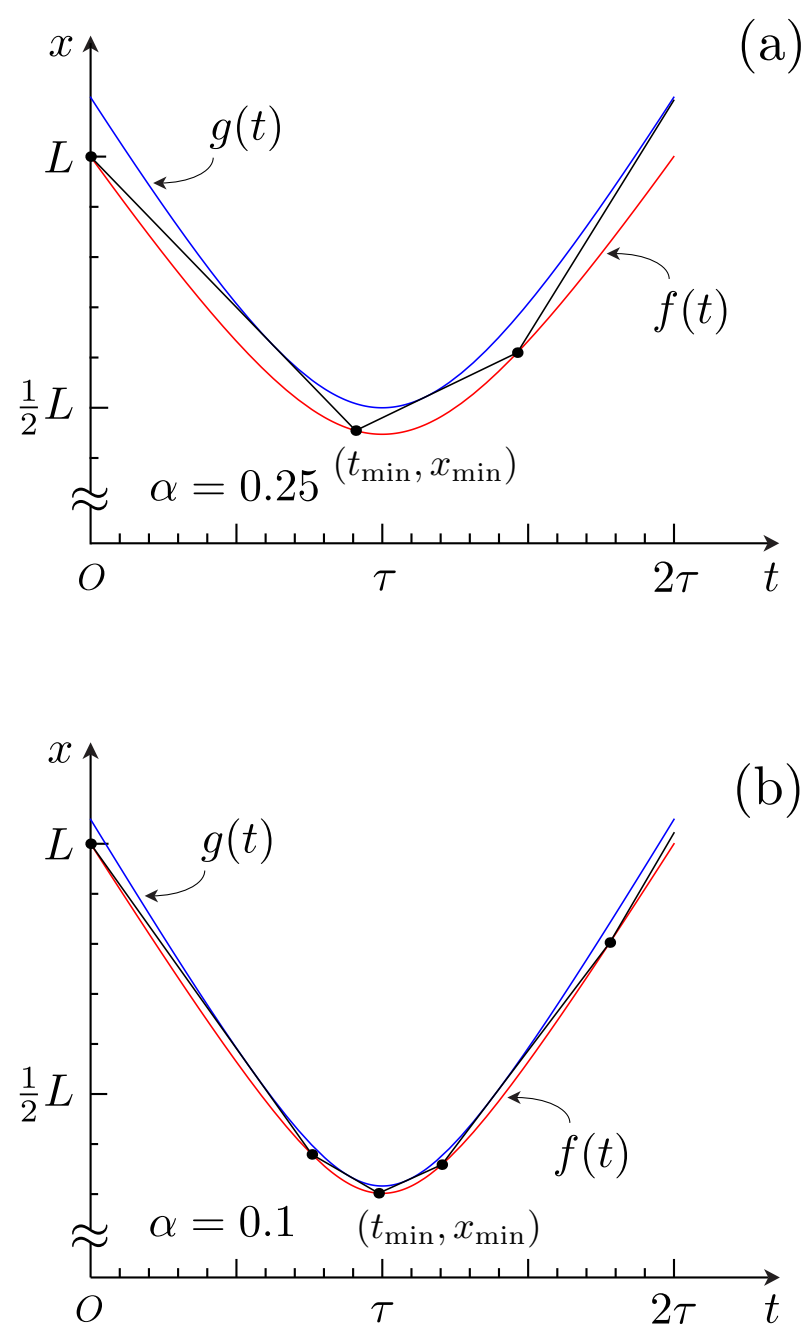

FIG. 3: (a) The collision points $P_{n}\left(t_{n}, x_{n}\right)$ at $\alpha=0.25$, where $N_{1}=2$ and $N=3$. The trajectory of the block $x(t)$ consists of line segments that connect every collision point $P_{n}$. The lower bound $f(t)$ passes every collision point $P_{n}$ and the upper bound $g(t)$ is tangent to every line segment $\overline{P_{n} P_{n+1}}$. The continuum representation $\bar{x}(t)$ of the trajectory is the arithmetic average of $g(t)$ and $f(t)$. (b) The same as (a) except that $\alpha=0.1, N_{1}=3$, and $N=5$. 